\title{
MAP LEGEND AND RESPONSE DESIGN: HOW DO THEY AFFECT ACCURACY OF GEOBIA RESULTS
}

\author{
J. Radoux ${ }^{\mathrm{a} *}$, P. Bogaert ${ }^{\mathrm{a}}$ \\ ${ }^{a}$ Earth and Life Institute, Université catholique de Louvain, 1348 Louvain-la-Neuve, Belgium - (julien.radoux,patrick.bogaert)@uclouvain.be
}

KEY WORDS: Accuracy assessment, quality, segmentation, legend, overall accuracy

\begin{abstract}
:
The legend used to map a given region is most of the time the first characteristic assessed by end users. In GEOBIA, the choice of a classification system is primarily guided by the scale of analysis and the underlying data model. This study focuses on the classification systems related to spatial regions. Depending on the purpose of the GEOBIA analysis, three types of classification systems have been identified: majority-based, LCCS like and feature-based. The validation of the classification results then relies on an appropriate choice of response design, which in turn influences the accuracy results. The impact of the different response designs is highlighted with a real case study. A high accuracy pixel-based classification of the segmented image is used as synthetic reference and classification errors are simulated on the object database. The results shows that all methods are equivalent if all polygons are pure, but that the uncertainty conferred by under-segmented or heterogeneous polygons affects the accuracy estimates in different ways: some methods tolerate delineation or under-segmentation while others are directly affected. This experiment also shows some differences and similarities between the point-based and the object-based validation frameworks, which are both valid depending on the circumstances but could be misleading when they are not applied with the right purpose. The thematic precision of the legend is also discussed: the LCCS authorize mixtures of different spatial entities in the class definition, but the information content differs depending of the purity of the majority of the classes.
\end{abstract}

\section{INTRODUCTION}

GEOBIA is now considered as a new image classification paradigm. It is therefore important to identify good practices for the quality assessment of GEOBIA results. As central part of the fitness to purpose assessment, the legend used to map a given region is most of the time the first characteristic considered by end users. In GEOBIA, the choice of a classification system is primarily guided by the scale of analysis and the underlying data model. Image-segments are indeed groups of pixels that may be used to represent spatial objects (which can be unambiguously delimited) or spatial regions (which do not have precise boundaries at the scale of the analysis and could be defined as a mixture of different entities). Classification systems for spatial objects usually imply a majority-based class assignment guided by pure reference objects. The spatial delineation of the image-segments is very important in this case. On the other hand, spatial regions are more often used to describe a continuous landscape with softer boundaries: in this case, partitioning classification systems like the LCCS should prevail while the positional errors along the boundary are usually not the primary quality issue.

This study focuses on the classification systems related to spatial regions. Depending on the purpose of the GEOBIA analysis, three types of classification systems have been identified: majority-based, LCCS (Di Gregorio and Jansen, 2000) like and feature-based. The validation of the classification results then relies on an appropriate choice of response design, which in turn influences the accuracy results. The impact of the different response designs is highlighted with a real case study. A high accuracy pixel-based classification of the segmented image is used as synthetic reference and classification errors are simulated on the object database. The results shows that all methods are equivalent if all polygons are pure, but that the uncertainty conferred by

\footnotetext{
${ }^{*}$ Corresponding author
}

under-segmented or heterogeneous polygons affects the accuracy estimates in different ways: some methods tolerate delineation or under-segmentation while others are directly affected. This experiment also shows some differences and similarities between the point-based and the object-based validation frameworks, which are both valid depending on the circumstances but could be misleading when they are not applied with the right purpose. The thematic precision of the legend is also discussed: the LCCS authorize mixtures of different spatial entities in the class definition, but the information content differs depending of the purity of the majority of the classes. Finally, it is shown that the accuracy of the LCCS-like classification system can be optimized for a given number of classes by selecting thresholds that minimize the confusion errors.

\section{METHOD}

A framework based on realistic synthetic data was set up in order to compare the different validation approaches. This is achieved by assuming that the delineation corresponds to the end user needs and using an existing classification as if it was the true land cover. Thematic classification errors are then simulated based on transposed confusion matrix of the classified map, but the delineation is not modified in order to avoid interactions between thematic and spatial errors. The study area could have been completely synthetic, but the use of a real world example allows us to better illustrate the discussion. This real world example uses a pixelbased classification and image segmentation based on the same data set. It covers the Walloon region at a resolution of 2 meters and the mean polygon size is around 6700 pixels with a minimum mapping unit of 1000 square meters.

\subsection{Classification systems}

The choice of a legend affects both the accuracy of a map and its fitness to purpose. Three classifications are used in this study: 
- Majority rule assigns a label based on the dominant land cover inside each polygon. This type of legend includes the minimum number of classes necessary to represent each of the basic classifiers. It is most suited when GEOBIA aims at identifying spatial object, which are pure with respect to the classifiers.

- FAO LCCS rules are built upon a set of thresholds on the proportion of each land cover within the spatial unit. When correctly applied, those rules generate a comprehensive partition of the feature space. They are well suited to coarse resolution (due to mixed pixels) or to the characterization of heterogeneous land cover classes based on spatial region. In this study, the LCCS rules used in the global land cover CCI project (Bontemps et al., 2013) have been applied. Considering the region, possible classes are 10, 30, 40, 60, 70, 90, 100, 110, 120, 190, 200 and 210. Those classes are characterized with an unique code that guarantees a good interoperability.

- In addition to those two expert-based rules, a third objectbased legend was driven by the data itself based on k-mean clustering. This legend is more difficult to interpret but the aim is to test its robustness relatively to expert-based legends.

Those labels were first assigned based on their reference values. In a second stage, random errors have been introduced into the pixel-based classification and labels were assigned on the altered data.

\section{RESPONSE DESIGNS AND SAMPLING UNITS}

Obviously, the ideal response design would consist in assigning a class to each object by measuring the proportion of each classifier and applying the legend rules. This method is however the most time consuming, which reduces the advantage of using polygonbased instead of point-based sampling (Radoux et al., 2011). The bias on the overall accuracy due to the sampling unit was therefore estimated for different alternative that could be applied on the field:

- Centroid-based sampling : similar sampling scheme to the standard probabilistic sampling, but polygons are selected from a list of all polygons then the point samples are defined as the centroids of each polygon.

- Parcel-based sampling: polygons centroids are first selected then the pixels in a circular plot around those centroids are used. In this study a 5 pixels radius $(10 \mathrm{~m})$ was arbitrarily selected. The different classification rules are then applied on the proportions of pixels belonging to each circular plot in order to determine their final label, which is compared with the polygon label.

In order to estimate the errors, ten random samples of 600 points were created using the centroids of randomly selected polygons. The values were then extracted from the unaltered pixel-based classification. Both classification accuracy (proportion of correctly classified polygons) and overall accuracy (proportion of the map area that is correctly classified) are then derived based on (Radoux and Bogaert, 2014).

\section{RESULTS}

The results show that the difference between a centroid-based interpretation and an object-based legend can be substantial. The bias of a single point sampling and the maximum or the LCCS object-based legends are $17.7 \%$ and $28.3 \%$, respectively. This bias is smaller with the parcel-based sampling, but it is still large: it reaches $11.5 \%$ for the majority label and $21.1 \%$ with the LCCS. As expected, the differences are linked with the heterogeneity of the spatial regions. Indeed, polygons that are close to purity (more than $90 \%$ of a single class) are matched with a simple point-based sampling in $98.3 \%$ of the cases. With parcelbased sampling, this value is even larger with $98.8 \%$. This suggest that centroid-based or parcel-based sampling unit could be used as a pragmatic alternative for the validation of spatial objects, but should be avoided with heterogeneous spatial regions. On the other hand, centroid-based sampling is not equiprobable because points along the object boundaries are less likely to be selected. Because object delineation errors are more likely around the edges, centroid-based classification overestimate the quality of spatial object classification. This issue can be solved by using standard probabilistic sampling or by assessing the delineation quality independently of the thematic accuracy.

\section{CONCLUSION}

This study demonstrates that the choice of sampling unit and response design have a major impact on the accuracy assessment results. Detailed results and discussion will be presented at the GEOBIA conference.

\section{ACKNOWLEDGEMENTS}

This study was supported by the Fédération Wallonie-Bruxelles in the frame of the Lifewatch-WB project.

\section{REFERENCES}

Bontemps, S., Defourny, P.and Radoux, J., Van Bogaert, E., Lamarche, C., Achard, F., Mayaux, P., Boettcher, M., Brockmann, C., Kirches, G., Zulkhe, M., Kalogirou, V., Seifert, F. M. and Arino, O., 2013. Consistent global land cover maps for climate modelling communities: Current achievements of the esa' land cover cci. ESA Living Planet Symposium, ESA-ESRIN, Frascati.

Di Gregorio, A. and Jansen, L., 2000. Land cover classification system (lccs): Classification concepts and user manual. GCP/RAF/287/ITA Africover-East Africa Project and Soil Resources, Management and Conservation Service, Food and Agriculture Organization.

Radoux, J. and Bogaert, P., 2014. Accounting for the area of polygon sampling units for the prediction of primary accuracy assessment indices. Remote Sensing of Environment 142, pp. 919.

Radoux, J., Bogaert, P., Fasbender, D. and Defourny, P., 2011. Thematic accuracy assessment of geographic object-based image classification. International Journal of Geographical Information Science 25(1), pp. 1365-8816. 